\title{
Pulse Contour Analysis for Prediction of Fluid Responsiveness in Preeclamptic Patients: A Case Series
}

\author{
Luciano Frassanito*1, Massimo Antonelli ${ }^{1}$, Antonio Messina ${ }^{2}$, Davide Colombo ${ }^{2}$ and Gaetano Draisci ${ }^{1}$ \\ ${ }^{1}$ Area Anestesiologia, Rianimazione, Terapie Intensive e Terapia del Dolore, Rome, Italy
}

${ }^{2}$ Department of Emergency Medicine, Ospedale Maggiore della Carità, Novara, Italy

Received: June 01, 2018; Published: June 12, 2018

*Corresponding author: Luciano Frassanito, Istituto di Anestesia e Rianimazione, Policlinico A. Gemelli Foundation, largo A. Gemelli 8, 00168 Rome, Italy

\begin{abstract}
Preeclampsia (PE) is a pregnancy-specific syndrome characterized by maternal cardiac output (CO) alterations and relative hypovolemia. In patients with PE is mandatory to predict Fluid Responsiveness (FR), above all if caesarean section (CS) under spinal anesthesia is required. The fluid challenge (FC) is a diagnostic approach to hemodynamic management which aims at identifying the patients who respond to fluid administration with an increase in CO. We present a case series to evaluate the effectiveness of a FC in increasing Cardiac Index (CI) in a group of preeclamptic patients using a continuous pulse-contour analysis. We studied the percentage of patients with PE scheduled for caesarean delivery who had a $15 \%$ increase of $\mathrm{CI}$ after a FC, in comparison with a control group of healthy pregnant patients. At baseline the patients in PE group showed higher Mean Arterial Pressure (MAP) and lower Stroke Volume Index (SVI). Fifteen patients were non responders (69\% of the patients in PE group and $46 \%$ of the patients in control). We suggest that the hemodynamic measurement of CI is useful to guide fluid therapy in pregnant woman with PE, and caution should be taken during fluid administration in all healthy pregnant woman at term.
\end{abstract}

Abbreviations: PE: Preeclampsia; CO: Cardiac Output; CS: Caesarean Section; FC: Fluid Challenge; CI: Cardiac Index; MAP: Mean Arterial Pressure; SVI: Stroke Volume Index; FR: Fluid Responsiveness; SV: Stroke Volume

\section{Introduction}

Preeclampsia (PE) is a pregnancy-specific multi-organ syndrome secondary to generalized vascular endothelial activation and vasospasm [1,2]. Maternal hemodynamic state is characterized by high vascular resistances, cardiac output (CO) alterations and relative hypovolemia [2]. Above all the women who develop "early onset" PE (time of the diagnosis at or before 34 weeks of gestational age) show evidence of left ventricular mild diastolic dysfunction and segmental impaired myocardial relaxation [2]. Several authors stated that assessment of maternal hemodynamic in women with $\mathrm{PE}$ is fundamental for appropriate fluid management $[3,4]$. Insufficient intravascular volume as fluid excess can lead to life-threatening scenarios, such as decreased oxygen delivery to tissues with multiorgan dysfunction, or fluid extravasation and pulmonary oedema [4]. Poor fluid management in PE has been reported as leading cause of admission to an intensive care unit and maternal deaths [3].

In these patients is mandatory to predict fluid responsiveness (FR), especially if caesarean section under spinal anesthesia is required, as adequate fluid therapy aimed at optimization of CO could be effective to prevent spinal induced hypotension and uteroplacental hypoperfusion [5]. Different studies suggested some strategies to evaluate fluid responsiveness [3,6]. but the debate about the best way to assess it is still open. The fluid challenge (FC) is a reliable diagnostic approach to hemodynamic management which aims at identifying the patients who respond to fluid administration with an increase in blood pressure or CO $[7,8]$. To our knowledge, FC with $\mathrm{CO}$ analysis was never tested in PE. We, therefore decided to evaluate if a FC might have the ability to predict FR in a group of preeclamptic patients using a continuous pulse-contour analysis.

\section{Patients}

After internal Medical Ethics Committee approval and collection of informed consent to analysis and publication of data from the women enrolled, we studied the percentage of patients with early-onset PE scheduled for caesarean delivery (13 patients, PE Group) who had a 15\% increase of Cardiac Index (CI) after a FC, in comparison with a control group of healthy pregnant patients (13 patients, CC Group) undergoing caesarean section for placenta previa. Non-invasive hemodynamic monitoring was performed in all patients as internal clinical practice in our centre. In PE group 
all the patients were treated with Metildopa $500 \mathrm{mg} 3$ times daily, plus Nifedipine $30 \mathrm{mg}$ twice a day for 2 cases. LiDCO plus monitor (LiDCO Ltd., Cambridge, UK) was used to calculate stroke volume (SV) from arterial waveform by autocorrelation algorithm [9].

Two consecutive calibrations with lithium chloride were used to convert nominal to actual SV and CO. We gave an arbitrary value of central venous pressure of $5 \mathrm{mmHg}$. We considered as baseline values the registration of one minute recorded three minutes after calibration of the monitor. The test of fluid responsiveness was carried out with Ringer Lactate solution $500 \mathrm{ml}$ infused within $5 \mathrm{~min}$. Data were elaborated with LiDCOviewTM program. The analysis of modification of hemodynamic variables minute to minute during FC was performed. Differences between the absolute value of variables within each group were studied, and baseline was chosen as control variable. For each hemodynamic variable the differences between PE and CC were evaluated by means of Unpaired t-test or Mann-
Whitney test when appropriate. The percentage of increase in CI, the difference between PE and CC and rate of early complication in both groups were evaluated.

\section{Results}

At baseline the patients in PE group showed higher mean arterial pressure and lower Stroke Volume Index, as compared to $\mathrm{CC}$ as showed in (Table1), but only the difference in MAP reached statistical significance. Overall 15 patients were not able to respond to FC with an increase in CI (non-responders, NR): 9 patients (69\%) were in PE group, while 6 (46\%) in CC group. Among responders the mean C.I. average $(\Delta \mathrm{CI})$ was $1.1+1.5 \mathrm{~L} / \mathrm{min} / \mathrm{m} 2$, SVI mean raise was $6.4+8.8 \mathrm{ml} / \mathrm{m} 2 /$ beat. No significant changes in HR, MAP and SVRI was recorded (Figure 1) shows the distribution of fluid responsiveness in the two groups: $\Delta \mathrm{CI}$ after $\mathrm{FC}$ was significantly lower in women with severe PE $(\mathrm{p}=0.002)$.

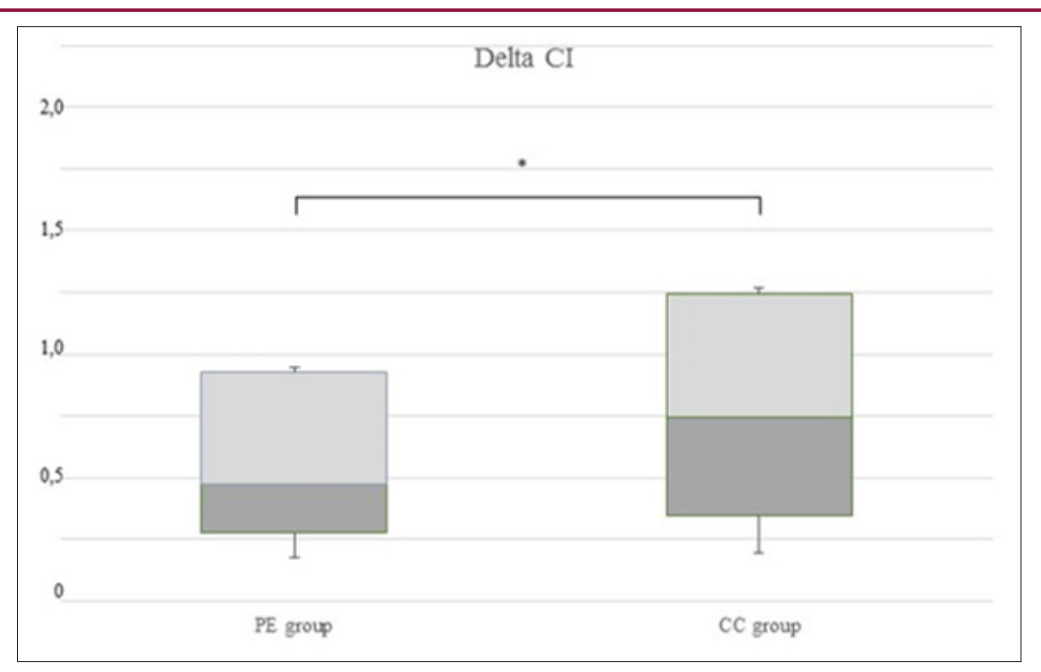

Figure 1:Box-and-whisker plot of Delta C.I., variation of cardiac index induced by fluid challenge (L/min/m2), in 13 women with severe preeclampsia (PE group) and in 13 healthy controls. Delta C.I. was higher in CC group than in PE group ( $\mathrm{p}=$ 0.002). ${ }^{*} \mathrm{p}<0.05$. Boxes with internal lines represent median and interquartile range and whiskers are range.

Table 1: Hemodynamic data of the two group of patients at baseline. The patients in PE group showed higher mean arterial pressure $(*=\mathrm{p}<0.05)$.

\begin{tabular}{|c|c|c|c|c|c|}
\hline Baseline & CI $\left(\mathrm{L} / \mathrm{min} / \mathrm{m}^{2}\right)$ & SVI (mL/m²/beat) & MAP(mmHg) & HR (beats/min) & SVRI(dynes-sec-5/m²) \\
\hline PE Group & $3.7 \pm 1.0$ & $38.4 \pm 14.1$ & $118 \pm 7$ & $90 \pm 14$ & $2609 \pm 741$ \\
\hline \multirow[t]{2}{*}{ CC Group } & $3.8 \pm 1.4$ & $48.0 \pm 9.3$ & $94 \pm 13$ & $78 \pm 20$ & $2084 \pm 929$ \\
\hline & $p=0.86$ & 0.07 & $0.002^{*}$ & 0.21 & 0.10 \\
\hline
\end{tabular}

\section{Discussion}

In normal pregnancy vascular dilation, reduction in arterial pressure and increased heart rate cause an increased CO. In PE, both a "hyperdynamic" state with high $\mathrm{CO}$ and low vascular resistance and a "hypodynamic" state with high resistances, low CO and low plasma volume are described $[1,2]$. Particularly the so called "earlyonset" PE (before 34 weeks of gestation) is characterized by high vascular resistance and low $\mathrm{CO}$, whereas "late onset" preeclampsia is dominated by high $\mathrm{CO}$ and low vascular resistance [1,2]. In early-onset preeclampsia, the endothelial dysfunction produces the haemodynamic changes, organ dysfunction and foetal growth restriction [1]. Several authors studied haemodynamic in PE using noninvasive monitoring tools, demonstrating these findings [2]. At our knowledge this is the first study to evaluate the ability of a FC to increase $\mathrm{CO}$ in spontaneously breathing patients affected by severe PE by a beat-to-beat analysis of SV. Our findings are in accordance with previous data $[2,3,10]$. Brun et al studied a population of 44 patients affected by severe PE and reported that only $52 \%$ of the patients who developed oliguria responded to a fluid challenge [3]. The authors outlined the poor predictive value of clinical features such as oliguria to assess fluid responsiveness and guide fluid management [3]. 
Ambrozic et al showed that severe PE is associated with ultrasound evidence of increase in extravascular lung water and disturbed diastolic left ventricular function compared with healthy pregnancies, and they found only a small proportion (about 14\%) of women with severe PE to be fluid responsive to passive leg raising [10]. Moreover, they observed a small percentage (67\%) of fluid responders to passive leg raising also among control group of healthy pregnant women at term. Nevertheless the compression of inferior vena cava by the gravid uterus in pregnant women at term, and the increased abdominal pressure make questionable the validity of passive leg raising in these clinical scenario, despite some authors argued in favor of it $[3,11]$. In our opinion is remarkable the finding that about one in two patients among healthy pregnant resulted NR to FC. A reduction in diastolic performance could be the main reason of these findings. Indeed Melchiorre et al. showed a tendency toward reduced diastolic reserve and impaired chamber diastolic function in a large population of pregnant women at term subjected to echocardiography [12]. A mild degree of diastolic dysfunction and segmental myocardial diastolic dysfunction were seen respectively in $17,9 \%$ and $28,4 \%$ of the women [12]. FC seems to be a reliable test that not requires specific expertise as compared to echocardiographic assessment. Moreover, it does not add invasively manoeuvre to PE normal management, but it allows a continuous monitoring that potentially might be useful to address and evaluate different therapeutic strategies according to fluid responsiveness. Further studies are required to evaluate the benefit of this monitoring in guide clinical decision. In conclusion we suggest that the hemodynamic measurement of CO and SV may be useful to guide fluid therapy in pregnant woman with SPE; moreover, caution should be taken during fluid administration in healthy pregnant woman at term undergoing to surgery.

\section{Acknowledgement}

Assistance with the study: We would like to thank Professor R. Proietti for his assistance with the study.

Financial support and sponsorship: This work was supported by the Department of Anaesthesiology and Intensive Care, Policlinico A. Gemelli Foundation, Rome.

\section{References}

1. Stocks G (2014) Preeclampsia: pathophysiology, old and new strategies for management. Eur J Anaesthesiol 31(4): 183-189.

2. Melchiorre K, Sharma R, Thilaganathan B (2014) Cardiovascular implications in preeclampsia: an overview. Circulation 130(8): 703-714.

3. Brun C, Zieleskiewicz L, Textoris J, Muller L, Bellefleur JP, et al. (2013) Prediction of fluid responsiveness in severe preeclamptic patients with oliguria. Intensive Care Med 39(4): 593-600.

4. Dennis AT, Solnordal CB (2012) Acute pulmonary oedema in pregnant women. Anaesthesia 67: 646-659.

5. Luo XJ, Zheng M, Tian G, Zhong HY, Zou XJ, et al. (2016) Comparison of the treatment effects of methoxamine and combining methoxamine with atropine infusion to maintain blood pressure during spinal anesthesia for cesarean delivery: a double blind randomized trial. Eur Rev Med Pharmacol Sci 20(3): 561-567.

6. Crozier TM, Wallace EM, Parkin WG (2015) Haemodynamic assessment in pregnancy and pre-eclampsia: A Guytonian approach. Pregnancy Hypertens 5(2): 177-181.

7. Messina A, Longhini F, Coppo C, Pagni A, Lungu R, et al. (2017) Use of the Fluid Challenge in Critically Ill Adult Patients: A Systematic Review. Anesth Analg 125(5): 1532-1543.

8. Guinot PG, Bernard E, Defrancq F, Petiot S, Majoub Y, et al. (2015) Mini-fluid challenge predicts fluid responsiveness during spontaneous breathing under spinal anaesthesia: An observational study. Eur J Anaesthesiol 32(9): 645-649.

9. Frassanito L, Vagnoni S, Zanfini BA, Catarci S, Maggiore S, et al. (2012) General anesthesia for caesarean delivery in a pregnant woman affected by acute myocardial infarction. Eur Rev Med Pharmacol Sci 16(8): 11231126.

10. Ambrozic J, Brzan Simenc G, Prokselj K, Tul N, Cvijic M, et al. (2017) Lung and cardiac ultrasound for hemodynamic monitoring of patients with severe pre-eclampsia. Ultrasound Obstet Gynecol. 49(1): 104-109.

11. Vårtun Å, Flo K, Acharya G (2014) Effect of passive leg raising on systemic hemodynamics of pregnant women: a dynamic assessment of maternal cardiovascular function at 22-24 weeks of gestation. PLoS One 9(4): e94629.

12. Melchiorre K, Sharma R, Khalil A, Thilaganathan B (2016) Maternal Cardiovascular Function in Normal Pregnancy: Evidence of Maladaptation to Chronic Volume Overload. Hypertension 67(4): 754762 .
Submission Link: https://biomedres.us/submit-manuscript.php

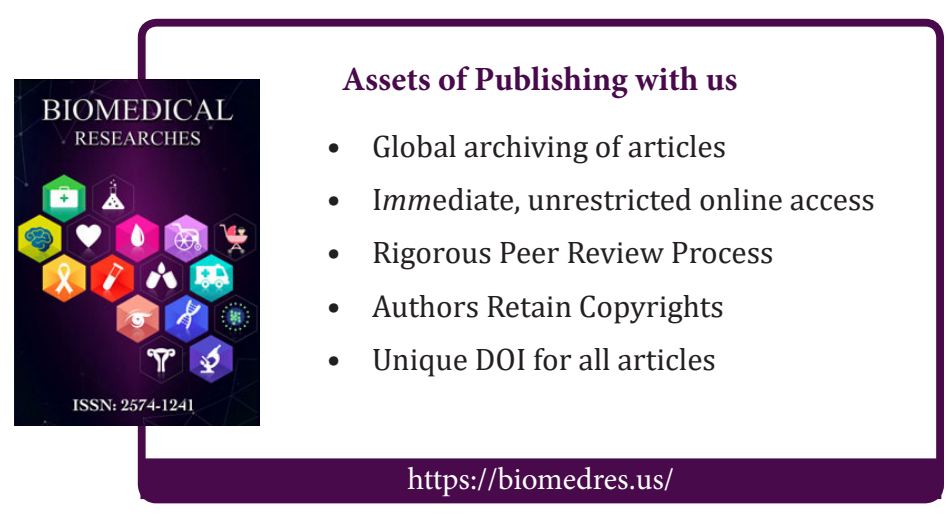

